\title{
Brush Swelling and Attachment Strength of Barnacle Adhesion Protein on Zwitterionic Polymer Films as a Function of Macromolecular Structure
}

\author{
Shifeng Guo, ${ }^{\dagger, \S}$ Robert Quintana, ${ }^{*},{ }^{\dagger, \ddagger}$ Marco Cirelli, ${ }^{\perp, \#}$ Zi Siang Desmond Toa, ${ }^{\dagger}$ (1) \\ Vivek Arjunan Vasantha, ${ }^{\oplus}$ E. Stefan Kooij, ${ }^{\nabla}$ Dominik Jańczewski, ${ }_{\odot}$ and G. Julius Vancso ${ }^{*}, \|, \perp_{\odot}$ \\ ${ }^{\dagger}$ Institute of Materials Research and Engineering A*STAR (Agency for Science, Technology and Research), Innovis, \#08-03, 2 \\ Fusionpolis Way, Singapore 138634 \\ ${ }^{\ddagger}$ Materials Research and Technology Department, Luxembourg Institute of Science and Technology (LIST), L-4422 Belvaux, \\ Luxembourg \\ ${ }^{\S}$ CAS Key Laboratory of Human-Machine Intelligence-Synergy Systems, Shenzhen Institutes of Advanced Technology, Chinese \\ Academy of Sciences, Shenzhen, Guangdong 518055, China \\ "Institute of Chemical and Engineering Sciences, A*STAR, 1 Pesek Road, Jurong Island, Singapore 627833 \\ ${ }^{\perp}$ Materials Science and Technology of Polymers, MESA+ Institute for Nanotechnology, "Faculty Engineering Technology, \\ Production Technology, and ${ }^{\nabla}$ Physics of Interfaces and Nanomaterials, MESA+ Institute for Nanotechnology, University of \\ Twente, P.O. Box 217, 7500 AE Enschede, The Netherlands \\ Faculty of Chemistry, Warsaw University of Technology, Noakowskiego 3, 00-664 Warsaw, Poland
}

\section{Supporting Information}

ABSTRACT: The exceptional hydration of sulfobetaine polymer brushes and their resistance toward nonspecific protein absorption allows for the construction of thin films with excellent antibiofouling properties. In this work, swollen sulfobetaine brushes, prepared by surface-initiated atom transfer radical polymerization of two monomers, differentiated by the nature of the polymerizable group, are studied and compared by a liquid-cell atomic force microscopy technique and spectroscopic ellipsometry. Colloidal AFMbased force spectroscopy is employed to estimate brush grafting density and characterize nanomechanical properties in salt water. When the ionic strength-induced swelling behaviors of the two systems are compared, the differences observed on the antipolyelectrolyte response can be correlated with the stiffness variation on brush compression, likely to be promoted by solvation differences. The higher solvation of amide groups is proposed to be responsible for the lower adhesion force of the barnacle cyprid's temporary adhesive proteins. The adhesion results provide further insights into the antibiofouling activity against barnacle cyprid settlement attributed to polysulfobetaine brushes.

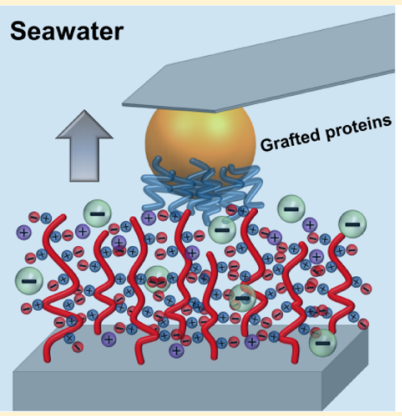

Barnacle cyprid footprint proteins

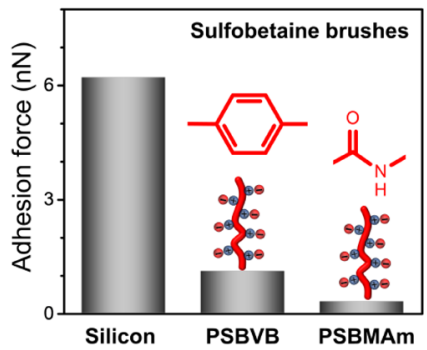

\section{INTRODUCTION}

Surfaces coated with zwitterionic polysulfobetaine-based brushes are good against biofouling and find an increasing research interest for applications, for example, in the biomedical $^{1-6}$ and maritime ${ }^{7-12}$ fields. The action mechanism for these materials is typically derived from the strong brush hydration and the formation of a tightly bonded and structured water layer along the polymer molecules. ${ }^{13-15}$ The most popular and studied example of this class of polymers is polysulfobetaine methacrylate (PSBMA). These brushes exhibit particularly high water uptakes and swelling responses, for instance, when compared with equivalent quaternized polyelectrolyte brushes. ${ }^{16,17}$ Swelling factors greater than 1 have been measured at relative humidity values above $20 \%{ }^{16}$
Both, PSBMA brushes' moisture uptake and wettability, are strongly governed by strong intermolecular and/or intramolecular dipolar interactions, which are linked to the high dipole moment of zwitterionic groups and the grafting density of the brush system. ${ }^{18}$ The occurrence and type of these dipolar interactions are considered to be responsible for brush wettability, which has been reported to be thickness- and temperature-dependent. ${ }^{19,20}$ The attractive electrostatic interactions, however, are screened in salt water due to the presence of free counterions in the solution. ${ }^{21}$ Recently, Takahara et al.

Received: March 29, 2019

Revised: May 15, 2019

Published: May 17, 2019 
a)
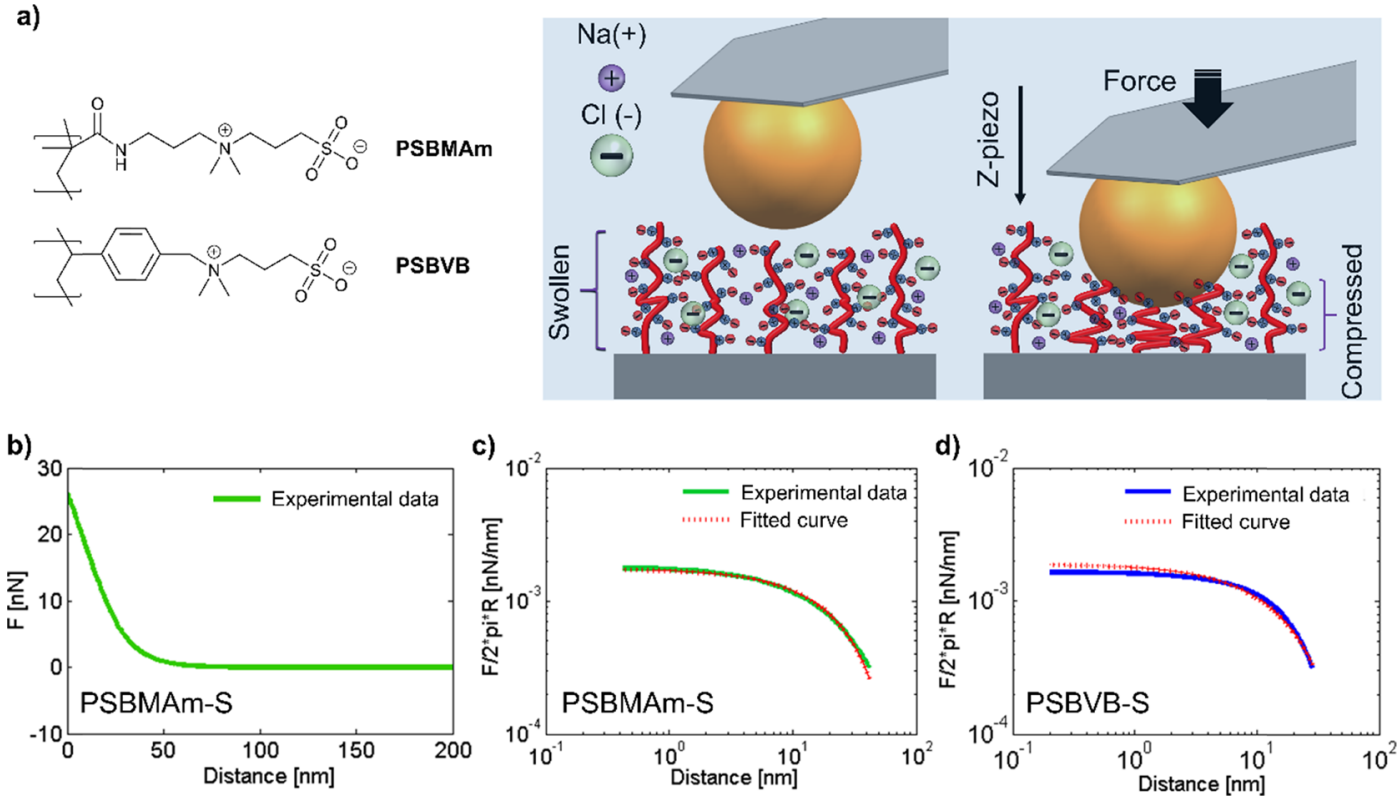

Figure 1. (a) Chemical structure of the brush monomers and schematics of the colloidal probe against the brush-coated surfaces, showing the interaction of the colloidal probe with the polymer brushes in salt water. (b) Representative AFM force-distance approach curves of the colloidal probe from the PSBMAm-S polymer brushes in $0.7 \mathrm{M} \mathrm{NaCl}$ solution. (c, d) Log-log plots, with the corresponding fitting according to eq 1, of the experimental data for PSBMAm-S and for PSBVB-S, respectively.

estimated the swollen thickness of PSBMA brushes on silicon surfaces as a function of salt concentration. ${ }^{22-25}$ They reported that PSBMA brush chains can form an extended conformation in salt water, while the chains shrink in pure water as a consequence of the strong attractive interaction between zwitterionic side groups. ${ }^{23}$ Indeed, zwitterionic polymers in aqueous media are frequently described as antipolyelectrolytes since increasing the ionic strength of their solution promotes swelling of polymer chains instead of collapse. ${ }^{26}$ However, not all zwitterionic brush systems show this effect. Kobayashi et al. investigated the response to changes in ionic strength of phosphorylcholine- and sulfobetaine-based methacrylic polymeric brush systems, PMPC and PSBMA, respectively, by varying $\mathrm{NaCl}$ concentration of aqueous solutions between 0 and $5 \mathrm{M} \mathrm{NaCl} .^{22,23}$ It was found that the thickness of the PMPC brush film was not salt concentration-dependent while PSBMA was greatly affected. A full understanding of the differences in physical properties and their relationship to molecular structure necessitates the employment of new experimental approaches, for example, the use of atomic force microscopy-based approaches.

Elasticity of a polymer brush (characterized by the system's Young's modulus) is an important physical characteristic that affects a system's performance in applications such as cell adhesion, ${ }^{27}$ brush biomembrane support and bending rigidity, ${ }^{28,29}$ microfluidics, ${ }^{30}$ and others. Atomic force microscopy (AFM) measurements have been used to determine the elastic properties of polymer brushes with thickness values up to hundreds of nanometers. ${ }^{31}$ AFM-based colloidal probe force measurements have been applied with success to determine the values of the compression modulus of zwitterionic polymer brushes. ${ }^{31,32}$ Modulus determination and compression experiments can also assist in characterizing the brush grafting density. Grafting density is a very important structural parameter of a polymer brush system. To estimate the grafting densities, some experimental methods were based on the assumption that chain length values of macromolecules grown in solution and from a substrate surface ${ }^{33}$ obtained under identical environmental conditions are the same, although simulation studies have questioned the validity of this statement. ${ }^{34}$ Hence, the precise determination of grafting density values remains a difficult task. ${ }^{35}$ The Alexander-de Gennes model can be used as a first approximation to correlate the height of a brush in a solvent with the grafting density and the degree of polymerization. ${ }^{32,36,37}$ The brush-brush interaction forces under compression measurements also provide insights into grafting densities. ${ }^{32,38,39}$

Polymeric brushes are used to reduce attachment of barnacle cyprids, ${ }^{5,40-42}$ which are considered as one of the most notorious fouling organisms due to their relative large adult size (reached at the end of the metamorphosis cycle) and ability to colonize nearly any man-made structure. The cyprids explore the surfaces of solids immersed in sea water to select the most suitable places to settle and secrete temporary adhesive proteins, which are deposited as "footprints" through the antennular attachment discs to attach themselves at the preferred locations. ${ }^{43}$ These proteins form temporary anchoring points for the migrating cyprids and can act as a settlement cue for other exploring cyprids. Although the morphology of footprint proteins has been explored using $\mathrm{AFM}^{44}$ the exact molecular and physiochemical nature of the footprint deposits remains largely unknown. It is worth noting that a settlementinducing protein complex (SIPC), which also functions as a settlement cue, has been described. ${ }^{45,46}$

Attachment strength is a key factor in the selection of the locations of attachment of barnacle cyprids during settlement, and one approach toward reducing the fouling impact of barnacles is to develop surfaces to which cyprid temporary and/or permanent adhesion is impossible. ${ }^{47}$ A quantitative assessment of interactions of cyprid footprint proteins with surfaces was achieved by colloidal probe-based AFM force spectroscopy. ${ }^{48,49}$ Briefly, the footprint proteins were covalently attached by chemical coupling to previously chemically activated colloids of AFM probes, and the adhesion strength of 
the proteins against the surfaces was measured. Recently, Shiomoto et al. immobilized live cyprids on AFM cantilevers, and the adhesion force of a cyprid's tentacles was determined by measuring the torsion of the cantilever when the tentacles were detached from glass surfaces coated with polymer brushes. $^{50}$

In our previous work, we reported on the active marine antifouling performance of three sulfobetaine-based systems. ${ }^{11}$ Bearing the same sulfobetaine group $\left(-\mathrm{NH}_{2}^{+}-\left(\mathrm{CH}_{2}\right)_{3}-\right.$ $\left.\mathrm{SO}_{3}{ }^{-}\right)$, the systems were characterized by the differences in linkage groups between the zwitterion and brush backbone. Limited differences in the settlement of the barnacle cyprid between the studied systems were observed. ${ }^{11}$ In this present work, by means of liquid AFM and complementary liquid ellipsometry measurements, the solvation of two of those sulfobetaine-based brush systems, namely, methacrylamide (PSBMAm) and vinylbenzene (PSBVB), was scrutinized in saline water. In addition, AFM-based force spectroscopy was implemented to estimate the grafting density of brush chains and compare the nanomechanical properties of the brush systems investigated. The results were then correlated with the brush swelling response affected by changes in ionic strength and temperature. Finally, aiming at associating brush hydration with their good marine antifouling performance, the adhesion force between cyprid footprint proteins and the brushmodified surfaces was investigated.

\section{EXPERIMENTAL SECTION}

Materials. Copper(I) chloride ( $\mathrm{CuCl}, 99.999 \%)$, copper(II) chloride $\left(\mathrm{CuCl}_{2}, 99.999 \%\right)$, tris(2-pyridylmethyl)amine (TPMA, 98\%), [3-(methacryloylamino)propyl]dimethyl-(3-sulfopropyl)-ammonium hydroxide inner salt (SBMAm, 96\%), and sodium chloride $(\mathrm{NaCl})$ were purchased from Sigma-Aldrich and were used without further purification. ( $p$-Chloromethyl)phenyl-trichlorosilane) (CMPS, $95 \%$ ) was purchased from Gelest, stored inside a nitrogen-filled glovebox, and used as received. SBVB was synthesized via quaternization of the tertiary amine precursor with sultone following reported protocol. ${ }^{11}$ All other chemicals were of reagent grade and were used without further purification. Deionized (DI) water (Millipore, $18 \mathrm{M} \Omega \cdot \mathrm{cm}$ ) and ultrapure nitrogen were used throughout. Silicon wafers with the thickness of $0.56 \mathrm{~mm}$ were purchased from Latech Scientific Supply Pte. Ltd. (Singapore).

Preparation of PSBMAm and PSBVB Brushes. The chemical structures of the SBMAm and SBVB monomers are shown in Figure 1a. Silicon wafers were exposed to the oxygen plasma for $5 \mathrm{~min}$ at 250 $\mathrm{W}$ using a microwave plasma chamber (Triple P, Duratek) in order to activate the surface. Subsequently, a CMPS ATRP initiator was deposited from the gas phase overnight and heated at $120{ }^{\circ} \mathrm{C}$ for 3 min in an oven. ${ }^{8}$ The initiator-modified silicon wafers were stored in a nitrogen-filled glovebox. All polymerizations were conducted at room temperature. In a general procedure, a Schlenk flask containing SBMAm $(34.2 \mathrm{~g}, 292 \mathrm{mmol})$ and $\mathrm{NaCl}(0.58 \mathrm{~g}, 1.0 \mathrm{mmol})$ was sealed with rubber septa and degassed by at least three vacuum nitrogen refill cycles. In a separate flask, $\mathrm{CuCl}(8.46 \mathrm{mg}, 0.086 \mathrm{mmol})$ was degassed in a similar process. To the flask containing the monomer and salt, a degassed mixture of $14.3 \mathrm{~mL}$ of TPMA stock solution $\left(8 \mu \mathrm{mol} \cdot \mathrm{mL}^{-1}\right.$ in DI water), $2 \mathrm{~mL}$ of $\mathrm{CuCl}_{2}$ stock solution $\left(14 \mu \mathrm{mol} \cdot \mathrm{mL}^{-1}\right.$ in DI water), and $3.75 \mathrm{~mL}$ of $\mathrm{DI}$ water was added under nitrogen protection, and the mixture was stirred until all solids were dissolved. This solution was then transferred by cannula to the flask containing the $\mathrm{CuCl}$ to obtain a pale green homogeneous solution after stirring. For kinetic studies, $2 \mathrm{~mL}$ of the reaction mixture was syringed under nitrogen protection into nitrogen-filled glass vials containing an ATRP initiator-modified silicon substrate each. After the polymerization, the substrates were washed with $0.5 \mathrm{M} \mathrm{NaCl}$ solution and subsequently washed overnight in warm DI water and finally dried under a stream of nitrogen. Following a similar procedure, surface-initiated atom transfer radical polymerization (SI-ATRP) of SBVB was carried out at a [SBVB]: [CuCl]: $\left[\mathrm{CuCl}_{2}\right]$ :[TPMA] molar feed ratio of 240:3:1:4 in $0.5 \mathrm{M} \mathrm{NaCl}$ solution at room temperature $15 \% \mathrm{w} / \mathrm{v}$ monomer concentration in solvent.

Measurement of Swollen Brush Thickness. A NanoWizard III instrument (JPK Instruments AG, Berlin, Germany) equipped with the NanoWizard head and controller was used in the AFM measurement. The thickness of polymer brushes represents a fundamental structural parameter of brush coatings and affects several properties of the polymer brushes such as the Young's modulus and hardness. In order to measure the thickness, the polymer brushes were gently scratched and locally removed off the substrate (silicon wafer) with a "blade" tip, and the brush thickness was then calculated from the cross-sectional analysis between the brush surface and the scratched areas. ${ }^{35}$ The thickness of the polymer brushes in the dry state was first investigated by tapping-mode AFM under ambient conditions using standard silicon probes $\left(k \approx 40 \mathrm{~N} \cdot \mathrm{m}^{-1}\right.$, Tap 300AL$\mathrm{G}$, budget sensors). The same regions were thereafter evaluated in Millipore water and $\mathrm{NaCl}$ solutions with progressively increasing ionic strength from $0.15,0.5,0.7,1$, to finally $5 \mathrm{M} \mathrm{NaCl}$. To change the solvent environment, the corresponding solution was gently injected into and retracted from the AFM liquid cell (approximately $1 \mathrm{~mL}$ ) using a different syringe, and a 15 min equilibration time was applied before the measurement. The brush thickness in the liquid was evaluated using the force imaging-based QI mode (Quantitative Imaging mode), which enables performing the measurement of polymer thickness at zero force, therefore minimizing compression of the polymer during measurement and reflecting the actual thickness in various ionic concentration stages. To minimize the compressibility effect, soft triangular-shaped silicon nitride cantilevers (Nano World, PNP-TR), with the spring constant in the range of $0.07-0.09 \mathrm{~N} \cdot \mathrm{m}^{-1}$, were employed. All thickness data were processed using the JPK data processing software (version 4.2).

Spectroscopic ellipsometry (SE) measurements were carried out on a variable-angle spectroscopic ellipsometer (VASE-32, J. A. Woollam Co., U.S.A.). Measurements of psi $(\Psi)$ and delta $(\Delta)$ were performed as a function of photon energy with a step size of $0.1 \mathrm{eV}$ in the range of $0.8-4.5 \mathrm{eV}$ (corresponding to a wavelength range of 1550-276 $\mathrm{nm}$ ) for the dry experiments and in the photon range of 1.1-4.5 eV (corresponding to a wavelength range of $1127-276 \mathrm{~nm}$ ) for the liquid experiments. The optical properties and the thickness of the zwitterionic polymer layers in the dry state were determined by performing the measurements at three different angles of incidence $\left(65^{\circ}, 70^{\circ}\right.$, and $\left.75^{\circ}\right)$ at room temperature and in air. Measurements in the aqueous environment were carried out using a custom-built liquid cell (approximately $150 \mathrm{~mL}$ ) with a fixed angle of incidence of $63^{\circ}$, already used in our previous works, ${ }^{51-54}$ and the temperature was controlled by an externally heated bath. Before the measurement of each sample, a calibration measurement was performed to assess the effect of the liquid cell on the optical path of the polarized light on the silicon sample in the dry state at $63^{\circ}$. The salt concentration was varied by adding to the liquid cell $5 \mathrm{M} \mathrm{NaCl}$ solution. The analysis of the brush layers was performed on the basis of the Cauchy dispersion relationship. In the Supporting Information, the application of the Cauchy model is described in detail. Dry and wet ellipsometry data were fitted in the wavelength range between 276 and $1250 \mathrm{~nm}$ and 300 and $900 \mathrm{~nm}$, respectively. In liquid media, the best fitting results were obtained considering a uniform and homogenous wet Cauchy layer model. Experiments were repeated on three different positions on the samples.

Grafting Density Characterization Using Colloidal AFMBased Force Spectroscopy. To estimate the grafting densities of the zwitterionic polymer systems, AFM-based force spectroscopy was employed, which has also been explored for other polymer systems $^{32,39,55}$ and is schematically shown in Figure $1 \mathrm{~b}$. The theoretical basis for all techniques to determine the force response of brushes has been provided by de Gennes. ${ }^{38}$ Following the original de Gennes model, the resulting force between a spherical body and grafted polymer brushes, with separation distances smaller than the 
radius of the colloidal probe, can be described with the following equation $(\text { eq } 1)^{32,39,55}$

$$
\frac{F(d)}{2 \pi R}=\frac{2 k_{\mathrm{B}} T L}{35} \Gamma^{3 / 2}\left[7\left(\frac{L}{d}\right)^{5 / 4}+5\left(\frac{d}{L}\right)^{7 / 4}-12\right]
$$

where $d$ is the surface separation distance, $F$ is the force acting between surfaces, $R$ is the radius of the probe, $L$ is the thickness of the polymer brush, and $\Gamma$ is the number of bound polymer chains per unit area (grafting density); $k_{\mathrm{B}}$ and $T$ are the Boltzmann constant and temperature. To analyze the grafting density of the two polymer brushes, PSBMAm and PSBVB, their equivalent structures with short brushes, that is, PSBMAm-S and PSBVB-S were evaluated in a $0.7 \mathrm{M}$ $\mathrm{NaCl}$ solution, while the polymer brushes are considered to be on the fully extended condition (see thickness data).

Elasticity Response (Apparent Young's Modulus) of Polymer Brushes by Colloidal AFM. The elasticity described by the apparent Young's modulus $\left(E_{\text {apparent }}\right)$ of polymer brushes was measured using colloidal contact AFM probes (with a $\mathrm{SiO}_{2}$ sphere with a diameter of $5 \mu \mathrm{m}$, NT-MDT, Russia) conducted in a liquid environment. The apparent elastic modulus $(E)$ of the polymer brushes were estimated from the nanoindentation curves as described elsewhere based on the Hertz model (eq 2) (11,32 $^{-10}$

$$
F=\frac{4 \sqrt{R_{\text {tip }}}}{3} \frac{E}{1-v^{2}} \delta^{3 / 2}
$$

where $F$ is the applied load, $R_{\text {tip }}$ is the radius of the colloidal probe $(2.50 \pm 0.05 \mu \mathrm{m}), v$ is the Poisson's ratio of the polymer films (assumed to be 0.5), $E$ is the apparent Young's modulus of polymer films, and $\delta$ is the deformation of the polymer films. To compare the elastic behavior of the PSBMAm and PSBVB, the Young's modulus of PSBMAm-S and PSBVB-S was evaluated in the $0.7 \mathrm{M} \mathrm{NaCl}$ solution, with the polymer brushes in fully extended condition (see thickness data).

Measurement of the Adhesion Force between Cyprid Footprint Proteins and Polymer Brushes. The adhesion force of barnacle cyprid proteins were measured following our published reports. ${ }^{47,48}$ In short, the colloidal AFM probes with a $\mathrm{SiO}_{2}$ sphere of $5 \mu \mathrm{m}$ in diameter (NT-MDT, Russia) were modified with cyprid footprint proteins using glutaraldehyde. Before and after the experiments, the spring constant of the cantilevers was calibrated using the thermal noise method $\left(0.07-0.1 \mathrm{~N} \cdot \mathrm{m}^{-1}\right) .{ }^{56}$ The modified probe was measured against the polymer brushes, and the adhesion forces were analyzed with the JPK data processing software (version 4.2). The force measurements were conducted in filtered sea water.

Barnacle Cyprid Culture. Barnacle cyprids of Amphibalanus amphitrite were cultured at the marine laboratory of the Tropical Marine Science Institute (TMSI), National University of Singapore. Two-day-old cyprids were used for the cyprid footprint protein adhesion analysis.

\section{RESULTS AND DISCUSSION}

Two series of sulfobetaine-based polymer brush thin films were prepared using only water as a solvent by SI-ATRP of the methacrylamide and the styrene-based monomers. By varying only the polymerization time, two different dry thicknesses, below $50 \mathrm{~nm}$ and above $100 \mathrm{~nm}$, were fabricated for each brush system, named $-\mathrm{S}$ and $-\mathrm{L}$, respectively. The measured thicknesses are summarized in Table 1, where an indication of their wet thicknesses in DI water, $1 \mathrm{M} \mathrm{NaCl}$ solution, and filtered seawater are presented as swelling ratios, defined as the quotient between wet $\left(d_{\text {wet }}\right)$ and dry $\left(d_{\text {dry }}\right)$ brush thickness values. The selection of $\mathrm{NaCl}$ aqueous solutions as a model to study ionic strength-induced swelling of our brush systems is a relevant choice if correlations with observations in marine environment are pursued. Seawater has a high salinity content $(\sim 600 \mathrm{mM})$ and features a mixture of several salts with sodium
Table 1. Water Contact Angle (WCA), Dry Thickness, and Swelling Ratio of Sulfobetaine-Based Brushes in $\mathrm{NaCl}$ Aqueous Solution and Filtered Seawater (FSW) Determined by AFM

\begin{tabular}{llcccc} 
& & & \multicolumn{3}{c}{ swelling ratio } \\
\cline { 4 - 6 } reference & WCA & $\begin{array}{c}\text { dry thickness(in } \\
\text { nanometers) }\end{array}$ & $\begin{array}{c}\text { DI } \\
\text { water }\end{array}$ & $\begin{array}{c}1 \mathrm{M} \\
\text { NaCl }\end{array}$ & FSW \\
PSBMAm-S & $14(2)$ & 43 & 1.3 & 4.1 & 4.7 \\
PSBMAm-L & $16(2)$ & 120 & 1.8 & 5.3 & 3.5 \\
PSBVB-S & $34(2)$ & 34 & 1.3 & 2.4 & 1.9 \\
PSBVB-L & $44(3)$ & 115 & 1.0 & 2.2 & 1.7 \\
PSBMA $^{22}$ & 11 & $\sim 30$ & 2.3 & 5 & \\
\hline
\end{tabular}

and chloride ions as the major constituents (approximately $85 \%)$. In order to determine swollen brush height values of our systems in the marine environment, measurements in $0.22 \mu \mathrm{m}$ filtered seawater (FSW) were conducted.

Grafting Density Characterization of the Polymer Systems. Grafting density is another key parameter to characterize polymer brushes in combination with the length (distribution) of polymer chains. In a good solvent, macromolecules in densely grafted polymer brushes stretch from the surface and extend due to an interplay and balance of solvent osmotic (enthalpy) and polymer entropic (orientation) free energy contributions. The grafting density values, as mentioned, were estimated using compression AFM measurements in aqueous solutions, with the polymer brushes assumed to be fully extended. ${ }^{32,39}$ As shown in the illustration of AFM force-distance curves registered to determine the grafting density, the representative force-distance approach curve from the PSBMAm-S polymer brush is shown in Figure $1 \mathrm{~b}$. The corresponding $F /(2 \pi R)$ values (see eq 1 ) as a function of estimated distance are shown in Figure 1c with fitting performed using eq 1 . The model assumes that the polymer brush is fully compressed; therefore, only the data with the applied forces above $5 \mathrm{nN}$ was used. The grafting density was estimated by performing a least-mean-square (LMS) fit for the model profiles to the experimental data. For each experiment, at least 100 force curves at different positions were analyzed, and the average grafting density $(\Gamma)$ for PSBMAm was determined to be $0.032 \pm 0.004$ chains $\cdot \mathrm{nm}^{-2}$, which is considered the same within error as the grafting density of PSBVB, $0.039 \pm 0.006$ chains $\cdot \mathrm{nm}^{-2}$.

Elasticity Characterization. The values of the apparent Young's modulus $(E)$ of the polymer brushes in the $1 \mathrm{M} \mathrm{NaCl}$ solvent condition were estimated by the Hertz model using AFM. ${ }^{31}$ Histograms $(N=50)$ of the distributions of $E$ values with the same grafting densities under the same liquid conditions are shown in Figure 2. The arithmetic mean values of $E$ of PSBMAm-S were estimated at $95 \pm 11 \mathrm{KPa}$, and the $E$ values of PSBVB were estimated at $349 \pm 61 \mathrm{KPa}$. Thus, the average stiffness of PSBVB is more than three times stiffer compared to that of the PSBMAm brush.

Swelling Behavior of the Polymer Brushes. Several structural parameters can govern the swelling of brush systems. Grafting density and brush chain length are considered to play the major role on swelling and its magnitude. ${ }^{57,58}$ As mentioned, the grafting density of the two systems studied here exhibited no significant difference. The swelling behavior of the PSBMAm and PSBVB brushes were studied by liquid AFM in the QI mode. The AFM cross-sectional profiles, as mentioned, provided the brush height values. ${ }^{31,35}$ The ionic 

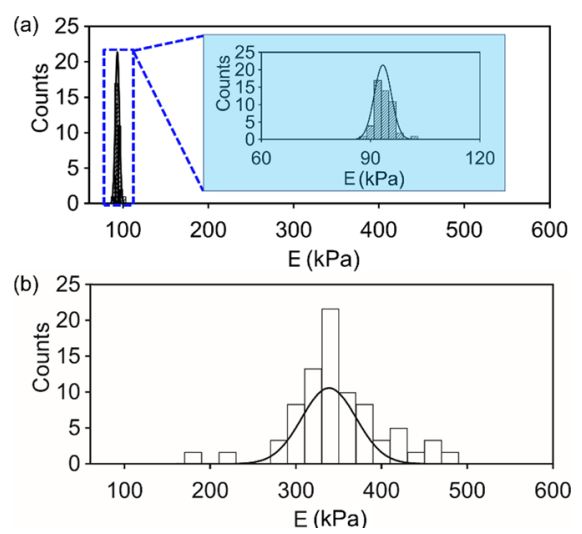

Figure 2. Statistical histograms of Young's modulus $(E)$ of polymer brushes in the salt solution at $1 \mathrm{M} \mathrm{NaCl}$ solution. (a) E distribution of PSBMAm-S; (b) E distribution of PSBVB-S.

strength of the liquid media varied from DI water to saturated $\mathrm{NaCl}$ solution concentrations. In all assays, the first and last measurements were carried out in DI water. Only differences within the standard deviation of the measurements were observed between these initial and final repeated measurements. As shown in Figure 3, both polymers were responsive to changes in ionic strength, but methacrylamide-based brush systems stretched significantly more than their styrene-based analogues. Considering the systems characterized by shorter brushes, PSBMAm-S and PSBVB-S, the swelling ratios in the absence of free counterions, that is, in DI water, are very similar with a value of approximately 1.3 . Similarly, the increase of $\mathrm{NaCl}$ concentration was followed by the increase of the swollen brush height, but the magnitude of the response was significantly different for the two systems. As an example, the swelling ratio of PSBMAm-S and PSBVB-S brushes in $1 \mathrm{M}$ $\mathrm{NaCl}$ solution was 4.1 and 2.4, respectively, clearly indicating a significant effect of counterions on the wet thickness.

Unavoidably, during liquid AFM measurements, the height of the measured polymer brushes may be affected by tip compression. To investigate the impact of this compression in the AFM measurements, complementary thickness data was obtained by in situ liquid spectroscopic ellipsometry $(\mathrm{SE})^{51,53}$ (see the Supporting Information for details on model selection and fitting procedure). Figure 4 shows the normalized thickness, calculated as swelling ratios, determined by AFM and $\mathrm{SE}$ in liquid media with salt concentrations up to $1 \mathrm{M}$ $\mathrm{NaCl}$. The data does not allow us to obtain a clear assessment. In DI water, AFM seems to underestimate the swollen thickness, and ellipsometry measurements exhibit lower swelling ratios with increasing ionic strength when the brush systems become more swollen. In this respect, it is reasonable to hypothesize that as brush swelling increases, the outer region of the brush film might become more diluted and more compressible, and eventually the position of the outermost brush interface within the pure liquid may be virtually impossible to observe. In such cases, ellipsometry will underestimate brush film thickness values and would be less sensitive to changes in ionic strength. Despite these uncertainties, in any case, both measurements indicated the higher swelling of the PSBMAm system.

The observed increase in wet thickness in both water and salt water does follow the observations of similar polyelectrolyte systems, which were found to not shrink at high salt concentrations as predicted by scaling laws. For instance, this antipolyelectrolyte phenomenon has been reported on PSBMA brushes with a similar dry thickness and slightly higher grafting density, $\sim 30 \mathrm{~nm}$ and 0.085 chains. $\mathrm{nm}^{-2}$, respectively. ${ }^{22}$ Its swelling data is included in Table 1 as a reference.

Following this comparison with the reported PSBMA brushes, a second series of brush systems characterized by longer brush length (PSBMAm-L and PSBVB-L) was studied to evaluate the effect of polymer chain length on the swelling response. ${ }^{19}$ However, contrary to the behavior reported for PSBMA systems with long brushes (i.e., $>80 \mathrm{~nm}$ ), which did not swell in DI water, ${ }^{19}$ PSBMAm-L stretched in a comparable manner, even slightly more than the shorter brushes. This trend was also observed when increasing the ionic strength. PSBVB-L, similar to PSBVB-S, appeared to be less responsive to the wet environment than PSBMAm brush systems. Indeed, the increase in polymer chain length reduced even their responsiveness with smaller changes in thickness. In this regard, PSBVB-L in DI water showed the same irresponsiveness, as reported for PSBMA systems of long brushes. Increasing the ionic strength was however followed by an
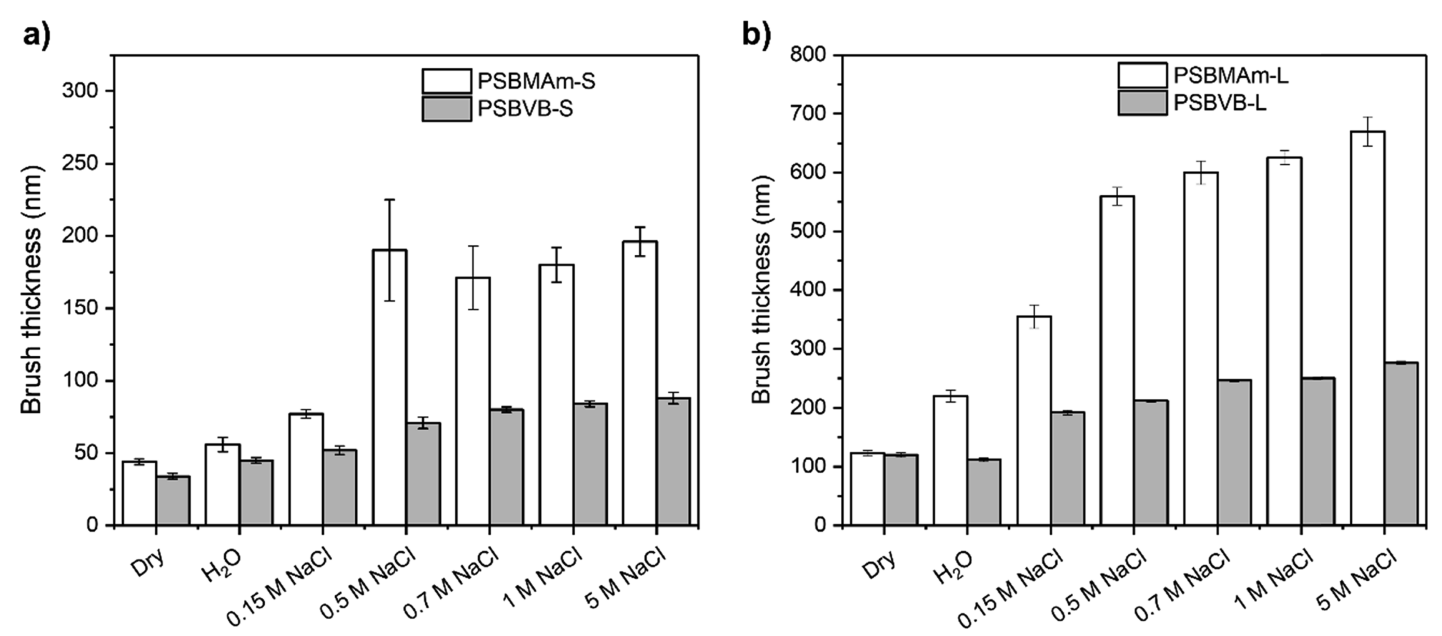

Figure 3. Swelling response of PSBMAm and PSBVB brush systems of different dry thicknesses varying the ionic strength $(\mathrm{NaCl}$ concn $)$ at $30{ }^{\circ} \mathrm{C}$ measured by AFM. 
a)

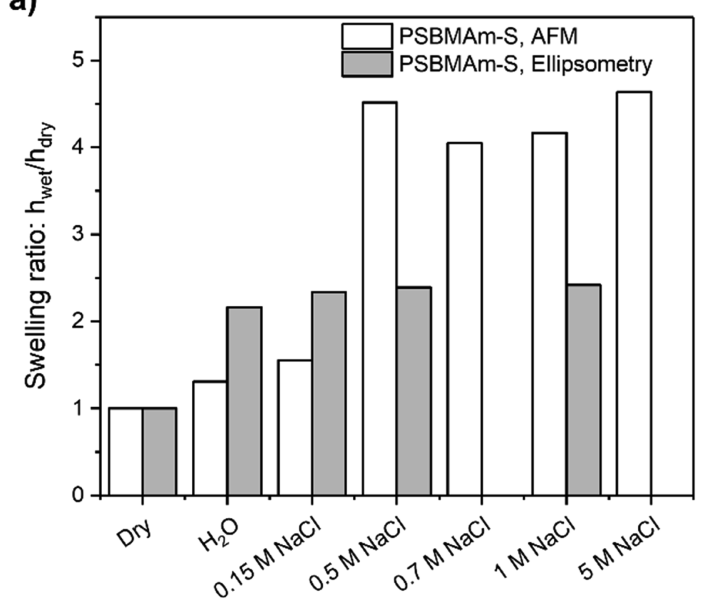

b)

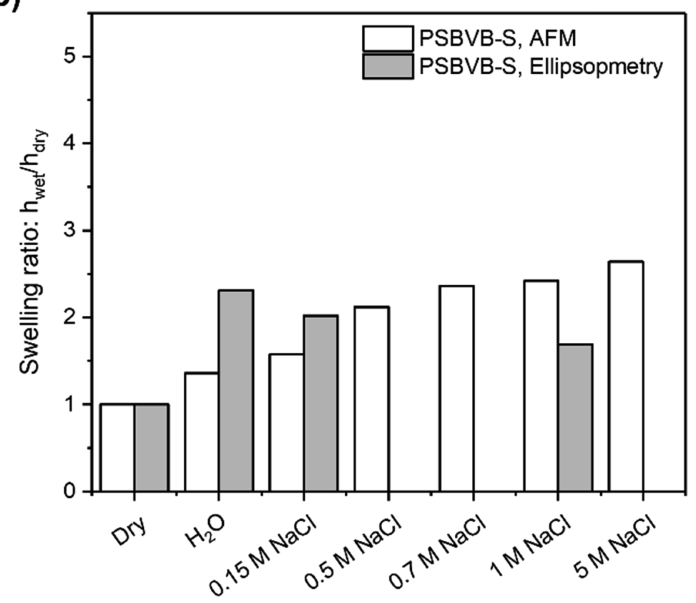

Figure 4. Swelling ratio (as histograms) calculated from the swollen height of the brushes and normalized by the dry thickness of the dry brush height as measured by AFM or by spectroscopic ellipsometry measurements for PSBMAm-S and PSBVB-S brush systems at $30^{\circ} \mathrm{C}$ as a function of $\mathrm{NaCl}$ concentration. Note: PSBVB-S was measured only by SE at $0,0.15$, and $1 \mathrm{M} \mathrm{NaCl}$.

increase of brush thickness, being just slightly less significant than that for PSBVB-S.

Solvation of zwitterionic brushes and the effect of zwitterionic groups on water hydrogen bonding network have been the subject of discussion in several recent publications. $^{20,22-24,59,60}$ Aiming at understanding the origin of the different swelling ratios observed on our two polymer brush systems and gathering further experimental results on their solvation mechanism, brush swelling measurements were conducted in water and deuterium oxide solutions. We suspected that replacing water with heavy water might affect brush hydration based on different hydrogen bonding formation between water and deuterium oxide molecules as the deuterium bond is about $5 \%$ stronger than the hydrogen bond. ${ }^{61}$ For this study, only PSBMAm-S and PSBVB-S were considered for the sake of simplicity. Comparison of swollen thicknesses of PSBVB and PSBMAm brushes in DI and deuterated water revealed that styrene-based brushes seemed not to be sensitive to changes of the isotope whether PSBMAm brushes stretch further in deuterated water, as shown in Figure 5. Interestingly, the presence of free counterions did not affect

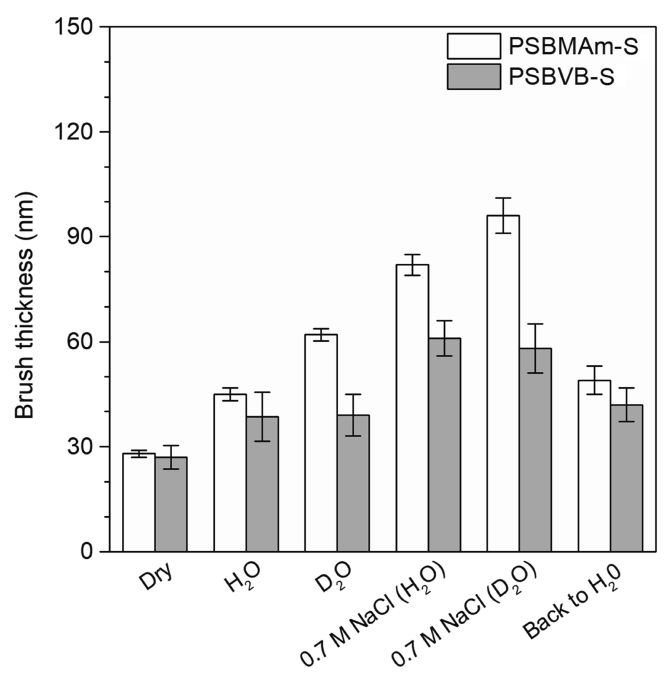

Figure 5. Swollen thickness determined by AFM of PSBMAm-S and PSBVB-S brushes in water and $0.7 \mathrm{M} \mathrm{NaCl}$. the trend showed by methacrylamide-based brushes. The observed difference in hydration suggests that deuterium oxide is a slightly better solvent for PSBMAm brushes than water. The cloud points of PSBMAm chains in solution have been reported to be higher in $\mathrm{D}_{2} \mathrm{O}$ than in DI water, independent of the molecular weight and polymer concentration. ${ }^{62}$ Since zwitterionic groups are considered equivalent in SBMAm and SBVB units, we interpret the observed brush hydration difference to be related to amide group hydrogen bonding formation and its effect on inducing brush swelling by solvation. Indeed, due to the amide group on SBMAm, this unit presents two more hydrogen bond acceptor/donor atoms than the SBVB unit, with only four bond acceptor/donor atoms. In this sense, studies of hydration properties of poly $(\mathrm{N}$ isopropylacrylamide) (PNIPAm) in water and deuterium dioxide by different methods have shown an increase of the average size of PNIPAm coils in $\mathrm{D}_{2} \mathrm{O}$ when compared with $\mathrm{H}_{2} \mathrm{O} .{ }^{63,64}$ Kujawa et al. considered that the difference in hydration might be related to higher organization of the $\mathrm{D}_{2} \mathrm{O}$ solution compared to $\mathrm{H}_{2} \mathrm{O} .{ }^{63}$ In our system, this hydration effect will increase the volume of water molecules directly interacting with the polymer chain, ${ }^{63}$ which might favor PSBMAm brush stretching. In a more recent work, Takahara and coworkers studied the swollen brush height of PSBMA brushes at different $\mathrm{NaCl}$ salt concentration in deuterium oxide using neutron reflectivity (NR) and AFM (DI water) measurements. $^{22}$ Their data suggests that no significant difference exists in brush height between water and deuterium oxide environments, independent of solution ionic strength, which is the same trend we observed for PSBVB brushes. It is worth noting, that ester groups on SBMA units are also capable of forming hydrogen bonds with water molecules. We note that recent simulation studies on the hydration of PSBMA and PSBMAm oligomers (16 units) in solution have shown only a slight increase in the number of water molecules in the first hydration shell on PSBMAm compared to PSBMA oligomers. $^{60}$

Polysulfobetaine brushes have been reported to exhibit upper critical solution temperature (UCST) properties. ${ }^{19,20}$ Huck et al. correlated the wetting transitions of PSBMA brushes observed upon UCST, characterized by an increase in hydrophilicity, with the change from a self-associated to a 


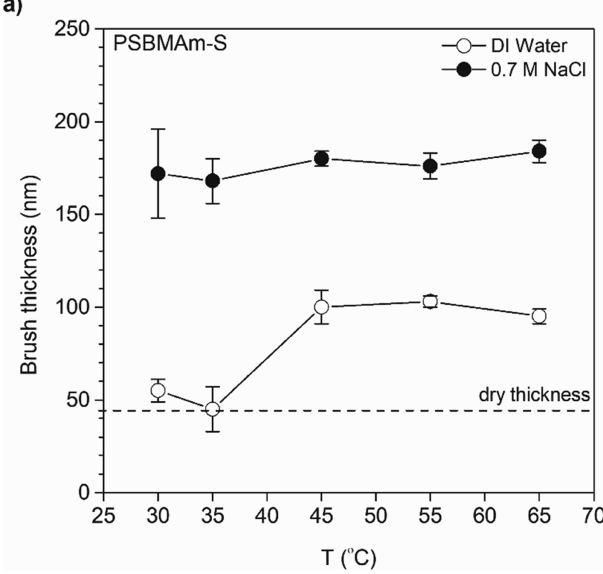

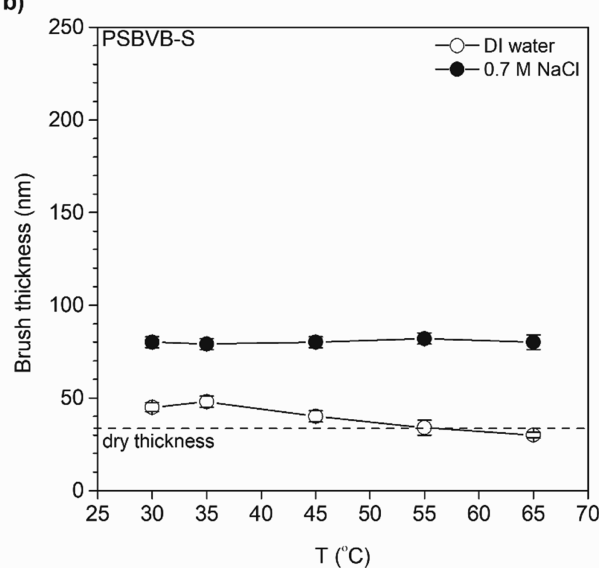

Figure 6. Swelling response of (a) PSBMAm-S and (b) PSBVB-S brush systems as a function of temperature in DI water and 0.7 M NaCl aqueous solutions determined by AFM.

nonassociated regime of the brush chains. ${ }^{19}$ In particular, the formation of a supercollapsed state below the UCST was proposed. This switching is considered to be linked with the disruption of the strong inter- and intrachain associations promoted by the zwitterionic moieties in water. ${ }^{19}$ In our work, the effect of temperature on the swelling response of the PSBMAm-S and PSBVB-S short brush systems was systematically evaluated in the range between 30 and $65{ }^{\circ} \mathrm{C}$ in $0.7 \mathrm{M}$ $\mathrm{NaCl}$ solution and DI water (Figure 6). With the exception of slight differences between PSBMAm and PSBVB systems, swollen brush height in salted water does not change significantly with temperature. However, the PSBMAm system showed an increase of swelling between 35 and $45{ }^{\circ} \mathrm{C}$ in DI water, without further evolution with temperature increase. This behavior is in agreement with the UCST properties reported for PSBMA brushes occurring at $40-50{ }^{\circ} \mathrm{C}$. ${ }^{19}$ Complementary SE measurements were carried out in DI water. However, taking into account the limitation of the experimental setup on variation and control of water temperature, the study was limited to two temperatures, that is, 25 and $50{ }^{\circ} \mathrm{C}$ (see the Supporting Information). The difference between swelling ratios at 50 and $25{ }^{\circ} \mathrm{C}$ for PSBMAm-S and PSBVB-S brushes amounted to 0.21 and 0.05 , respectively. This result indicates an increase of swelling for PSBMAm-S at $50{ }^{\circ} \mathrm{C}$ and almost no change in swollen height of the PSBVB-S brush system. Despite the fact that the values determined by SE are smaller than those obtained by AFM, the difference observed in responsiveness to changes in temperature between brush systems is preserved. We interpret our results as improvement of PSBMAm brush hydration due to the increase of water temperature. ${ }^{20}$ Considering dipolar interactions, an increase of temperature might break them and promote brush expansion due to solvation enhancement. In the presence of a high concentration of free counterions, this effect might be concealed by the major swelling promoted by the screening of the zwitterionic groups. Oppositely, PSBVB system results might not indicate the presence of UCST. Hence, once again, the difference in hydration properties of PSBVB and PSBMAm brush systems is indicated. Regarding the wettability of the brush systems, as expected for sulfobetaine-based brushes, all the surfaces were hydrophilic as shown by water contact angles at room temperature, below $45^{\circ}$ for styrene brushes and approximately $15^{\circ}$ for the methacrylamide systems. The differences between water contact angle values can be reasonably correlated with the higher hydrophobicity of the styrene backbone.

Adhesion of Cyprid Footprint Proteins. Colloidal AFMbased force spectroscopy was used to measure and compare the adhesion forces between the cyprid temporary adhesive proteins and the polymers, as described earlier in detail. ${ }^{48,49}$ In short, the cyprid footprint proteins secreted by the exploring cyprid was first visualized in filtered seawater conditions using AFM, and the cyprid footprint proteins were subsequently immobilized onto the glutaraldehyde-functionalized colloidal probe. The aldehyde groups at the colloidal probe surface form imine links to primary amine groups of the protein, which ensures a stable covalent attachment and no protein detachment during measurement. ${ }^{49,65,66}$ The adhesion force between cyprid footprint proteins and surfaces was compared among the surfaces.

Significant differences in adhesion forces were observed among the different surfaces. For the silicon control surface, the significantly higher adhesion force was attributed to the opposite charge of the proteins and surfaces in seawater condition. ${ }^{49}$ With a significantly lower adhesion value than the result on control silicon, the footprint proteins show stronger adhesion on the PSBVB-S polymer than on PSBMAm-S. The average adhesive force of footprint proteins with PSBVB is 1.1 $\pm 0.3 \mathrm{nN}$, which is 3 times larger than the values obtained from the PSBMAm, with the value of $0.3 \pm 0.1 \mathrm{nN}$ (Figure 7a). Laboratory cyprid settlement tests were previously conducted to evaluate the antifouling performance of similar surfaces. ${ }^{11}$ The cyprid preference differs significantly over the control and polymer brush-covered surfaces. More than $50 \%$ of the settlement was observed on the control surfaces, while only about $10 \%$ of cyprids settled on the PSBVB and PSBMAm surfaces (Figure $7 \mathrm{~b}$ ). Footprint adhesion results, in this case, correlate well with our previous cyprid settlement data, and it is in agreement with other reported data on sulfobetaine polymer brushes. ${ }^{7}$ Attachment strength is considered as a key factor in the selection of surfaces by barnacle cyprids during settlement, and one approach toward reducing the fouling impact of barnacles is to develop surfaces to which temporary and/or permanent adhesion by the cyprid is greatly reduced, or even impossible. ${ }^{47}$ To achieve efficient fouling prevention, a material needs to prevent the initial cyprid attachment and minimize the adhesion strength when a fouler encounters the surface. ${ }^{67}$ Our results suggest that the interaction between the 

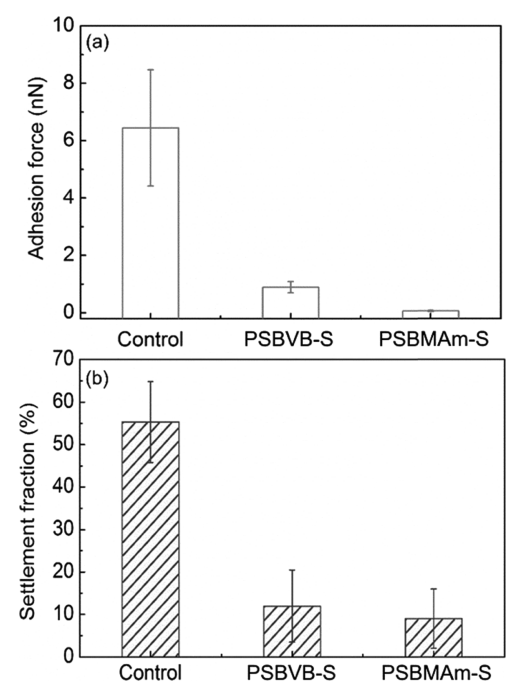

Figure 7. (a) Comparison of adhesion forces on different surfaces in filtered seawater and (b) comparison with the settlement behavior of cyprids on the surfaces. ${ }^{11}$ Unmodified silicon surfaces were used as control.

settlement adhesion proteins and the negatively charged silicon surfaces was much stronger than with zwitterionic surfaces due to the high solvation of the polymer brushes. Surface Young's modulus values affect cyprid settlement, and it was reported that surfaces with higher elastic modulus values were found to accumulate more fouling and exhibit poorer fouling release properties. ${ }^{68}$ Zwitterionic brushes are known to prevent barnacle settlement due to alteration of surface exploration behavior of barnacle cyprids. ${ }^{7}$ It has been reported that cyprids expend more time exploring the surface modified with sulfobetaine brushes than on unmodified bare glass. ${ }^{7}$ These results sustain the concept that footprint-surface interaction affects cyprid exploratory behavior and thus settlement. Electrostatic interactions play an important role in adhesion phenomena particularly for biomacromolecules and microorganisms.

\section{CONCLUSIONS}

The hydration of thin and thick polymer brush systems consisting of different zwitterionic polymers, that is, PSBMAm and PSBVB, differing in the chemical structure of the linking group between the backbone and the dipolar unit, was prepared by SI-ATRP of monomers in full aqueous media. The grafting density and Young's modulus values of the brushes studied were estimated by colloidal AFM-based force spectroscopy. Grafting densities were found to be very similar between systems while the styrene-based brushes were significantly stiffer than the methacrylamide-based brushes. AFM measurements in $\mathrm{H}_{2} \mathrm{O}$ and $\mathrm{D}_{2} \mathrm{O}$ were used to study the swelling responses and link it to the changes in the media. The observed phenomena are a consequence of the increase of solvation of methacrylamide-based brushes promoted by hydrogen bond formation with water molecules. The combination of zwitterionic moieties and amide groups, considered as hydrogen bonding promoters, within the same side group is responsible for thermoresponsive behavior which dominates the brush conformation in aqueous media. This result highlights the need for considering the linkage group between the zwitterion and brush chain in order to understand the solvation of sulfobetaine-based brushes. In high-ionic- strength media such as marine salt water, the stronger hydration of methacrylamide-based brushes is considered to be responsible for the lower adhesion force of cyprid footprint proteins to the surface. We demonstrate the suitability of AFM colloidal probes for the study of hydration and protein antiadhesion properties of antifouling polymer brushes.

\section{ASSOCIATED CONTENT}

\section{S Supporting Information}

The Supporting Information is available free of charge on the ACS Publications website at DOI: 10.1021/acs.langmuir.9b00918.

In situ liquid ellipsometry, thermoresponsive behavior and the effect of $\mathrm{NaCl}$ concentration on the PSBVB-S and the PSBMAm-S brush layers, comparison between uniform and homogenous Cauchy layer models, and a linear A-graded Cauchy layer model for the analysis of the wet measurements (PDF)

\section{AUTHOR INFORMATION}

\section{Corresponding Authors}

*E-mail: roberto.quintana@list.lu (R.Q.).

*E-mail: g.j.vancso@utwente.nl (G.J.V.).

\section{ORCID $\odot$}

Robert Quintana: 0000-0003-0879-053X

Zi Siang Desmond Toa: 0000-0003-2890-6579

Vivek Arjunan Vasantha: 0000-0001-7304-3261

Dominik Jańczewski: 0000-0002-5466-6444

G. Julius Vancso: 0000-0003-4718-0507

\section{Author Contributions}

All authors contributed to the writing of this manuscript. All authors have given approval for publication of the final version.

\section{Notes}

The authors declare no competing financial interest.

\section{ACKNOWLEDGMENTS}

The authors are grateful to the Agency for Science, Technology and Research (A*STAR) for providing financial support under the Innovative Marine Antifouling Solutions (IMAS) program. S.G. thanks the financial support from the Science and Technology Innovation Commission of Shenzhen (grant no. JCYJ20180507182239617). M.C. thanks the Marie Curie Initial Training Network "Complex Wetting Phenomena" for the financial assistance within the CoWet grant, agreement no. 607861. We acknowledge the University of Twente and its Mesa+ Research Institute for Nanotechnology for the additional assistance.

\section{REFERENCES}

(1) Cheng, G.; Zhang, Z.; Chen, S.; Bryers, J. D.; Jiang, S. Inhibition of bacterial adhesion and biofilm formation on zwitterionic surfaces. Biomaterials 2007, 28, 4192-4199.

(2) Smith, R. S.; Zhang, Z.; Bouchard, M.; Li, J.; Lapp, H. S.; Brotske, G. R.; Lucchino, D. L.; Weaver, D.; Roth, L. A.; Coury, A.; Biggerstaff, J.; Sukavaneshvar, S.; Langer, R.; Loose, C. Vascular catheters with a nonleaching poly-sulfobetaine surface modification reduce thrombus formation and microbial attachment. Sci. Transl. Med. 2012, 4, 153ra132.

(3) Chang, Y.; Chang, Y.; Higuchi, A.; Shih, Y. J.; Li, P. T.; Chen, W. Y.; Tsai, E. M.; Hsiue, G. H. Bioadhesive control of plasma proteins and blood cells from umbilical cord blood onto the interface grafted with zwitterionic polymer brushes. Langmuir 2012, 28, 4309-17. 
(4) Chang, Y.; Liao, S. C.; Higuchi, A.; Ruaan, R. C.; Chu, C. W.; Chen, W. Y. A Highly Stable Nonbiofouling Surface with Well-Packed Grafted Zwitterionic Polysulfobetaine for Plasma Protein Repulsion. Langmuir 2008, 24, 5453-5458.

(5) Sin, M. C.; Sun, Y. M.; Chang, Y. Zwitterionic-based stainless steel with well-defined polysulfobetaine brushes for general bioadhesive control. ACS Appl. Mater. Interfaces 2013, 6, 861-73.

(6) Dundua, A.; Franzka, S.; Ulbricht, M. Improved Antifouling Properties of Polydimethylsiloxane Films via Formation of Polysiloxane/Polyzwitterion Interpenetrating Networks. Macromol. Rapid Commun. 2016, 37, 2030-2036.

(7) Aldred, N.; Li, G.; Gao, Y.; Clare, A. S.; Jiang, S. Modulation of barnacle (Balanus amphitrite Darwin) cyprid settlement behavior by sulfobetaine and carboxybetaine methacrylate polymer coatings. Biofouling 2010, 26, 673-83.

(8) Zhang, Z.; Finlay, J. A.; Wang, L.; Gao, Y.; Callow, J. A.; Callow, M. E.; Jiang, S. Polysulfobetaine-Grafted Surfaces as Environmentally Benign Ultralow Fouling Marine Coatings. Langmuir 2009, 25, 13516-13521.

(9) Yang, W. J.; Neoh, K.-G.; Kang, E.-T.; Teo, S. L.-M.; Rittschof, D. Polymer brush coatings for combating marine biofouling. Prog. Polym. Sci. 2014, 39, 1017-1042.

(10) Guo, S.; Jańczewski, D.; Zhu, X.; Quintana, R.; He, T.; Neoh, K. G. Surface charge control for zwitterionic polymer brushes: Tailoring surface properties to antifouling applications. J. Colloid Interface Sci. 2015, 452, 43-53.

(11) Quintana, R.; Jańczewski, D.; Vasantha, V. A.; Jana, S.; Lee, S. S. C.; Parra-Velandia, F. J.; Guo, S.; Parthiban, A.; Teo, S. L.-M.; Vancso, G. J. Sulfobetaine-based polymer brushes in marine environment: Is there an effect of the polymerizable group on the antifouling performance? Colloids Surf., B 2014, 120, 118-124.

(12) Dębowski, M. A.; Quintana, R.; Lee, H. P. Compact test apparatus for evaluation of flow erosion of marine coatings. Rev Sci Instrum. 2015, 86, 105115.

(13) He, Y.; Hower, J.; Chen, S.; Bernards, M. T.; Chang, Y.; Jiang, S. Molecular Simulation Studies of Protein Interactions with Zwitterionic Phosphorylcholine Self-Assembled Monolayers in the Presence of Water. Langmuir 2008, 24, 10358-10364.

(14) Chen, S.; Li, L.; Zhao, C.; Zheng, J. Surface hydration: Principles and applications toward low-fouling/nonfouling biomaterials. Polymer 2010, 51, 5283-5293.

(15) Schlenoff, J. B. Zwitteration: coating surfaces with zwitterionic functionality to reduce nonspecific adsorption. Langmuir 2014, 30, 9625-36.

(16) Galvin, C. J.; Dimitriou, M. D.; Satija, S. K.; Genzer, J. Swelling of polyelectrolyte and polyzwitterion brushes by humid vapors. J. Am. Chem. Soc. 2014, 136, 12737-45.

(17) Galvin, C. J.; Genzer, J. Swelling of Hydrophilic Polymer Brushes by Water and Alcohol Vapors. Macromolecules 2016, 49, 4316-4329.

(18) Bredas, J. L.; Chance, R. R.; Silbey, R. Head-head interactions in zwitterionic associating polymers. Macromolecules 1988, 21, 16331639.

(19) Azzaroni, O.; Brown, A. A.; Huck, W. T. S. UCST wetting transitions of polyzwitterionic brushes driven by self-association. Angew. Chem., Int. Ed. 2006, 45, 1770-4.

(20) Cheng, N.; Brown, A. A.; Azzaroni, O.; Huck, W. T. S. Thickness-Dependent Properties of Polyzwitterionic Brushes. Macromolecules 2008, 41, 6317-6321.

(21) Lowe, A. B.; McCormick, C. L. Synthesis and Solution Properties of Zwitterionic Polymers. Chem. Rev. 2002, 102, 41774190.

(22) Kobayashi, M.; Terayama, Y.; Kikuchi, M.; Takahara, A. Chain dimensions and surface characterization of superhydrophilic polymer brushes with zwitterion side groups. Soft Matter 2013, 9, 5138.

(23) Terayama, Y.; Kikuchi, M.; Kobayashi, M.; Hino, M.; Takahara, A. Influence of salt concentration on swelling states of poly(sulfobetaine) brush at aqueous solution interface. J. Phys.: Conf. Ser. 2009, 184, No. 012011.
(24) Kobayashi, M.; Mitamura, K.; Terada, M.; Yamada, N. L.; Takahara, A. Characterization of Swollen States of Polyelectrolyte Brushes in Salt Solution by Neutron Reflectivity. J. Phys.: Conf. Ser. 2011, 272, No. 012019.

(25) Kikuchi, M.; Terayama, Y.; Ishikawa, T.; Hoshino, T.; Kobayashi, M.; Ogawa, H.; Masunaga, H.; Koike, J.-i.; Horigome, M.; Ishihara, K.; Takahara, A. Chain dimension of polyampholytes in solution and immobilized brush states. Polym. J. 2012, 44, 121-130.

(26) Schulz, D. N.; Peiffer, D. G.; Agarwal, P. K.; Larabee, J.; Kaladas, J. J.; Soni, L.; Handwerker, B.; Garner, R. T. Phase behaviour and solution properties of sulphobetaine polymers. Polymer 1986, 27, $1734-1742$.

(27) Mizutani, A.; Kikuchi, A.; Yamato, M.; Kanazawa, H.; Okano, T. Preparation of thermoresponsive polymer brush surfaces and their interaction with cells. Biomaterials 2008, 29, 2073-2081.

(28) Evans, E.; Rawicz, W. Elasticity of "Fuzzy" Biomembranes. Phys. Rev. Lett. 1997, 79, 2379-2382.

(29) Chen, Q.; Vancso, G. J. pH Dependent Elasticity of Polystyrene-block-poly(acrylic acid) Vesicle Shell Membranes by Atomic Force Microscopy. Macromol. Rapid Commun. 2011, 32, 1704-1709.

(30) Uhlmann, P.; Merlitz, H.; Sommer, J. U.; Stamm, M. Polymer brushes for surface tuning. Macromol. Rapid Commun. 2009, 30, 73240.

(31) Sui, X.; Chen, Q.; Hempenius, M. A.; Vancso, G. J. Probing the collapse dynamics of poly $(\mathrm{N}$-isopropylacrylamide) brushes by AFM: effects of co-nonsolvency and grafting densities. Small 2011, 7, 14407.

(32) Kutnyanszky, E.; Vancso, G. J. Nanomechanical properties of polymer brushes by colloidal AFM probes. Eur. Polym. J. 2012, 48, 815.

(33) Matyjaszewski, K.; Dong, H.; Jakubowski, W.; Pietrasik, J.; Kusumo, A. Grafting from surfaces for "everyone": ARGET ATRP in the presence of air. Langmuir 2007, 23, 4528-31.

(34) Turgman-Cohen, S.; Genzer, J. Simultaneous bulk- and surfaceinitiated controlled radical polymerization from planar substrates. $J$. Am. Chem. Soc. 2011, 133, 17567-9.

(35) Sui, X.; Zapotoczny, S.; Benetti, E. M.; Schön, P.; Vancso, G. J. Characterization and molecular engineering of surface-grafted polymer brushes across the length scales by atomic force microscopy. J. Mater. Chem. 2010, 20, 4981.

(36) Butt, H.-J.; Kappl, M.; Mueller, H.; Raiteri, R.; Meyer, W.; Rühe, J. Steric Forces Measured with the Atomic Force Microscope at Various Temperatures. Langmuir 1999, 15, 2559-2565.

(37) Cuenot, S.; Gabriel, S.; Jérôme, R.; Jérôme, C.; Fustin, C.-A.; Jonas, A. M.; Duwez, A.-S. First Insights into Electrografted Polymers by AFM-Based Force Spectroscopy. Macromolecules 2006, 39, 84288433.

(38) de Gennes, P. G. Conformations of Polymers Attached to an Interface. Macromolecules 1980, 13, 1069-1075.

(39) Block, S.; Helm, C. A. Conformation of Poly(styrene sulfonate) Layers Physisorbed from High Salt Solution Studied by Force Measurements on Two Different Length Scales. J. Phys. Chem. B 2008, 112, 9318-9327.

(40) Brzozowska, A. M.; Parra-Velandia, F. J.; Quintana, R.; Xiaoying, Z.; Lee, S. S. C.; Chin-Sing, L.; Jańczewski, D.; Teo, S. L.-M.; Vancso, J. G. Biomimicking micropatterned surfaces and their effect on marine biofouling. Langmuir 2014, 30, 9165-75.

(41) Yang, W. J.; Cai, T.; Neoh, K. G.; Kang, E. T.; Dickinson, G. H.; Teo, S. L.-M.; Rittschof, D. Biomimetic anchors for antifouling and antibacterial polymer brushes on stainless steel. Langmuir 2011, 27, 7065-76.

(42) Higaki, Y.; Nishida, J.; Takenaka, A.; Yoshimatsu, R.; Kobayashi, M.; Takahara, A. Versatile inhibition of marine organism settlement by zwitterionic polymer brushes. Polym. J. 2015, 47, 811.

(43) Yule, A. B.; Walker, G. Settlement of Balanus Balanoides: The Effect of Cyprid Antennular Secretion. J. Mar. Biol. Assoc. U.K. 1985, 65, 707. 
(44) Phang, I. Y.; Aldred, N.; Ling, X. Y.; Huskens, J.; Clare, A. S.; Vancso, G. J. Atomic force microscopy of the morphology and mechanical behaviour of barnacle cyprid footprint proteins at the nanoscale. J. R. Soc., Interface 2010, 7, 285-96.

(45) Matsumurad, K.; Nagano, M.; Kato-Yoshinaga, Y.; Yamazaki, M.; Clare, A. S.; Fusetani, N. Immunological studies on the settlement-inducing protein complex (SIPC) of the barnacle Balanus amphitrite and its possible involvement in larva-larva interactions. Proc. R. Soc. B. 1998, 265, 1825-30.

(46) Dreanno, C.; Matsumura, K.; Dohmae, N.; Takio, K.; Hirota, H.; Kirby, R. R.; Clare, A. S. An $\alpha_{2}$-macroglobulin-like protein is the cue to gregarious settlement of the barnacle Balanus amphitrite. Proc. Natl. Acad. Sci. U. S. A. 2006, 103, 14396-401.

(47) Aldred, N.; Scardino, A.; Cavaco, A.; de Nys, R.; Clare, A. S. Attachment strength is a key factor in the selection of surfaces by barnacle cyprids (Balanus amphitrite) during settlement. Biofouling 2010, 26, 287-299.

(48) Guo, S.; Puniredd, S. R.; Jańczewski, D.; Lee, S. S. C.; Teo, S. L. M.; He, T.; Zhu, X.; Vancso, G. J. Barnacle larvae exploring surfaces with variable hydrophilicity: influence of morphology and adhesion of "footprint" proteins by AFM. ACS Appl. Mater. Interfaces 2014, 6, 13667-76.

(49) Guo, S.; Zhu, X.; Jańczewski, D.; Lee, S. S. C.; He, T.; Teo, S. L. M.; Vancso, G. J. Measuring protein isoelectric points by AFMbased force spectroscopy using trace amounts of sample. Nat. Nanotechnol. 2016, 11, 817-23.

(50) Shiomoto, S.; Yamaguchi, Y.; Yamaguchi, K.; Nogata, Y.; Kobayashi, M. Adhesion force measurement of live cypris tentacles by scanning probe microscopy in seawater. Polym. J. 2019, 51, 51-59.

(51) Yu, Y.; Cirelli, M.; Kieviet, B. D.; Kooij, E. S.; Vancso, G. J.; de Beer, S. Tunable friction by employment of co-non-solvency of PNIPAM brushes. Polymer 2016, 102, 372-378.

(52) Ramakrishna, S. N.; Cirelli, M.; Kooij, E. S.; Klein Gunnewiek, M.; Benetti, E. M. Amplified responsiveness of multilayered polymer grafts: synergy between brushes and hydrogels. Macromolecules 2015, $48,7106-7116$.

(53) Kooij, E. S.; Sui, X.; Hempenius, M. A.; Zandvliet, H. J.; Vancso, G. J. Probing the thermal collapse of poly (Nisopropylacrylamide) grafts by quantitative in situ ellipsometry. $J$. Phys. Chem. B 2012, 116, 9261-9268.

(54) Sui, X.; Di Luca, A.; Gunnewiek, M. K.; Kooij, E. S.; van Blitterswijk, C. A.; Moroni, L.; Hempenius, M. A.; Vancso, G. J. Stability and cell adhesion properties of poly ( $\mathrm{N}$-isopropylacrylamide) brushes with variable grafting densities. Aust. J. Chem. 2011, 64, 1261-1268.

(55) Wang, J.; Butt, H. J. Forces between Thiolate-Modified Gold Surfaces in a Melt of End-Functionalized Polymers. J. Phys. Chem. B 2008, 112, 2001-2007.

(56) Hutter, J. L.; Bechhoefer, J. Calibration of atomic-force microscope tips. Rev. Sci. Instrum. 1993, 64, 1868-1873.

(57) Schüwer, N.; Klok, H. A. Tuning the $\mathrm{pH}$ sensitivity of poly(methacrylic acid) brushes. Langmuir 2011, 27, 4789-96.

(58) Chen, Q.; Kooij, E. S.; Sui, X.; Padberg, C. J.; Hempenius, M. A.; Schön, P. M.; Vancso, G. J. Collapse from the top: brushes of poly(N-isopropylacrylamide) in co-nonsolvent mixtures. Soft Matter 2014, 10, 3134-42.

(59) Murakami, D.; Kobayashi, M.; Moriwaki, T.; Ikemoto, Y.; Jinnai, H.; Takahara, A. Spreading and structuring of water on superhydrophilic polyelectrolyte brush surfaces. Langmuir 2013, 29, $1148-51$.

(60) Zhao, C.; Zhao, J.; Li, X.; Wu, J.; Chen, S.; Chen, Q.; Wang, Q.; Gong, X.; Li, L.; Zheng, J. Probing structure-antifouling activity relationships of polyacrylamides and polyacrylates. Biomaterials 2013, 34, 4714-4724.

(61) Némethy, G.; Scheraga, H. A. Structure of Water and Hydrophobic Bonding in Proteins. IV. The Thermodynamic Properties of Liquid Deuterium Oxide. J. Chem. Phys. 1964, 41, 680-689.

(62) Hildebrand, V.; Laschewsky, A.; Zehm, D. On the hydrophilicity of polyzwitterion poly (N,N-dimethyl-N-(3- (methacrylamido)propyl)ammoniopropane sulfonate) in water, deuterated water, and aqueous salt solutions. J. Biomater. Sci., Polym. Ed. 2014, 25, 1602-18.

(63) Kujawa, P.; Winnik, F. M. Volumetric Studies of Aqueous Polymer Solutions Using Pressure Perturbation Calorimetry: A New Look at the Temperature-Induced Phase Transition of Poly $(\mathrm{N}$ isopropylacrylamide) in Water and D2O. Macromolecules 2001, 34, 4130-4135.

(64) Wang, X.; Wu, C. Light-Scattering Study of Coil-to-Globule Transition of a Poly(N-isopropylacrylamide) Chain in Deuterated Water. Macromolecules 1999, 32, 4299-4301.

(65) Xu, L. C.; Siedlecki, C. A. Effects of surface wettability and contact time on protein adhesion to biomaterial surfaces. Biomaterials 2007, 28, 3273-83.

(66) Barattin, R.; Voyer, N. Chemical modifications of AFM tips for the study of molecular recognition events. Chem. Commun. 2008, 1513-32.

(67) Lejars, M.; Margaillan, A.; Bressy, C. Fouling Release Coatings: A Nontoxic Alternative to Biocidal Antifouling Coatings. Chem. Rev. 2012, 112, 4347-4390.

(68) Brzozowska, A. M.; Maassen, S.; Goh Zhi Rong, R.; Benke, P. I.; Lim, C. S.; Marzinelli, E. M.; Jańczewski, D.; Teo, S. L.-M.; Vancso, G. J. Effect of Variations in Micropatterns and Surface Modulus on Marine Fouling of Engineering Polymers. ACS Appl. Mater. Interfaces 2017, 9, 17508-17516. 